\title{
Quasi-stellar objects acting as potential strong gravitational lenses in the SDSS-III BOSS survey
}

\author{
Romain A. Meyer ${ }^{1,2}$, Timothée Delubac ${ }^{1}$, Jean-Paul Kneib ${ }^{1,3}$, and Frédéric Courbin ${ }^{1}$ \\ ${ }^{1}$ Institute of Physics, Laboratory of Astrophysics, Ecole Polytechnique Fédérale de Lausanne, 1015 Lausanne, Switzerland \\ 2 Department of Physics and Astronomy, University College London, Gower Street, London WC1E 6BT, UK \\ e-mail: r.meyer.17@ucl.ac.uk \\ 3 Aix Marseille Université, CNRS, LAM (Laboratoire d'Astrophysique de Marseille), UMR 7326, 13388 Marseille, France
}

Received 24 December 2018 / Accepted 11 March 2019

\section{ABSTRACT}

\begin{abstract}
We present a sample of 12 quasi-stellar objects (QSOs) that potentially act as strong gravitational lenses on background emission line galaxies (ELG) or Lyman- $\alpha$ emitters (LAEs) selected through a systematic search of the 297301 QSOs in the Sloan Digital Sky Survey (SDSS)-III Data Release 12. Candidates were identified by looking for compound spectra, where emission lines at a redshift larger than that of the quasar can be identified in the residuals after a QSO spectral template is subtracted from the observed spectra. The narrow diameter of BOSS fibers $\left(2^{\prime \prime}\right)$ then ensures that the object responsible for the additional emission lines must lie close to the line of sight of the QSO and hence provides a high probability of lensing. Among the 12 candidates identified, nine have definite evidence for the presence of a background ELG identified by at least four higher-redshift nebular emission lines. The remaining three probable candidates present a strong asymmetrical emission line attributed to a background Lyman- $\alpha$ emitter (LAE). The QSO-ELG (QSO-LAE) lens candidates have QSO lens redshifts in the range $0.24 \lesssim z_{\text {QSO }} \lesssim 0.66\left(0.75 \lesssim z_{\text {QSO }} \lesssim 1.23\right)$ and background galaxy redshifts in the range $0.48 \lesssim z_{\mathrm{S} \text {,ELG }} \lesssim 0.94\left(2.17 \lesssim z_{\mathrm{S}, \mathrm{LAE}} \lesssim 4.48\right)$. We show that the algorithmic search is complete at $>90 \%$ for QSO-ELG systems, whereas it falls at $40-60 \%$ for QSO-LAE, depending on the redshift of the source. Upon confirmation of the lensing nature of the systems, this sample may quadruple the number of known QSOs acting as strong lenses. We have determined the completeness of our search, which allows future studies to compute lensing probabilities of galaxies by QSOs and differentiate between different QSO models. Future imaging of the full sample and lens modelling offers a unique approach to study and constrain key properties of QSOs.
\end{abstract}

Key words. gravitational lensing: strong - methods: data analysis - surveys - quasars: general

\section{Introduction}

As gravitational lensing produces unmistakably distorted, amplified and multiplied images of the lensed objects, it is not surprising that the first gravitational lenses were discovered by identifying multiply imaged bright sources such as quasi-stellar objects (QSOs; e.g. Walsh et al. 1979; Weymann et al. 1980; Young et al. 1981; Huchra et al. 1985). Since the first discoveries, the increasing number of wide-field surveys has revealed the use, first suggested by Zwicky (1937), of strong gravitational lensing as a powerful tool to weigh individual galaxies and probe their radial mass profile (e.g Warren \& Dye 2003; Wayth et al. 2005; Bolton et al. 2012). Large samples of sourceselected lenses are now available thanks to the work of multiple teams over the last two decades (e.g. Kochanek et al. 1995; Muñoz et al. 1998; Browne et al. 2003; Myers et al. 2003; Oguri et al. 2006, 2008; Cabanac et al. 2007; Faure et al. 2008; More et al. 2011, 2016a; Inada et al. 2012; Williams et al. 2018; Agnello et al. 2018). Recent studies search for strong lenses through careful processing of large imaging datasets (e.g Joseph et al. 2014; Paraficz et al. 2016; Ostrovski et al. 2017) or through citizen science projects such as Spacewarps (More et al. 2016b)

Whereas source-selected samples span a wide range of physical properties of the lenses, lens-selected samples enable us to study specifically the targeted lenses. The largest lens-selected sample available to date is the Sloan Lens ACS Survey (SLACS, Bolton et al. 2006, 2008; Auger et al. 2009), where the lenses are early-type galaxies with redshift $0.16<z<0.49$ selected from the Sloan Digital Sky Survey (SDSS). The SLACS survey uses spectroscopic data to search for extra emission lines superimposed on the foreground galaxy spectra, following the method of Warren et al. (1996).

Motivated by the success of SLACS (Bolton et al. 2006; Shu et al. 2017), the Optimal Line-of-Sight lens survey (OLSlens survey; Willis et al. 2006), the BOSS Emission Line Lens Survey (BELLS; Brownstein et al. 2012), the SLACS for The Masses Survey (S4TM; Shu et al. 2015), the BELLS GALaxyLyman- $\alpha$ EmitteRs Systems survey (BELLS GALLERY; Shu et al. 2016a,b) and the subsequent confirmation of the majority of its Galaxy-LAE candidates (Cornachione et al. 2018), we decided to explore the potential role of QSOs as lenses further.

In light of these previous works, we believe that both lens- and source-selected strong lenses samples can now be obtained from wide-field spectroscopic surveys, when the foreground object is easily identified. Selection of the lensed object is enabled by the detection of specific emission lines such as Lyman- $\alpha$ or the presence of both [OII] $\lambda 3727 \AA, \mathrm{H} \beta$, [OIII] $\lambda 4959 \AA$, [OIII] $\lambda 5007 \AA$ and/or H $\alpha$ suggesting the presence of higher redshift Lyman- $\alpha$ Emitters (LAE) and emissionline galaxies, respectively.

Miralda-Escudé \& Léhar (1992) argued that galaxy-galaxy strong lenses should be ubiquitous, and the above surveys have overcome the challenge of their detection. However, it is 
not the same for QSO-galaxy lenses. In a pioneering study, Courbin et al. (2010) identify 14 QSOs acting as potential strong gravitational lenses and confirmed three of them (Courbin et al. 2012) using the Hubble Space Telescope (HST) deep imaging capacities (programme GO\#12233, Wide Field Camera 3 and UVIS detector). The research was conducted over SDSSII Data Release 7, fitting and subtracting a spline QSO continuum before cross-correlating the residuals with appropriate emission line templates. However, the small number of confirmed QSO lenses limits the analysis to the intrinsic properties of the individual targets. The promise of a statistically significant sample of QSO lenses is to compare their dynamical and lensing mass distribution and test the scaling laws between the QSO emission lines, the black hole mass and the host galaxy total mass (e.g. Kaspi et al. 2005, Shen et al. 2008). In a more recent study, Cen \& Safarzadeh (2017) have shown that strong lensing by QSOs could act as an efficient test of different models of dark matter halos of QSO host galaxies (Shen et al. 2013; Cen \& Safarzadeh 2015). With our new sample, we can increase the number of known QSO lenses by up to a factor of three or four and open the door to streamlined detection of such objects in future wide-field surveys. This would in turn benefit studies linking QSOs to their host galaxies.

In this paper, we present 12 new QSOs potentially acting as strong lenses in the SDSS-III BOSS Data Release 12 (DR12; Eisenstein et al. 2011; Dawson et al. 2013; Alam et al. 2015; Smee et al. 2013) as well as our selection method which extends spectroscopic selection of strong lenses to foreground QSOs. In Sect. 2, we review our candidate-selection method and provide spectroscopic evidence for all 12 candidate systems, as well as photometric hints for probable lensing features for one QSO-ELG lens candidate. In Sect. 3.1, we show the evidence for the first nine candidates being probable QSO-ELG strong lensing systems presenting higher redshift OII, $\mathrm{H} \beta$ and OIII on top of their QSO spectrum. The remaining three candidates in Sect. 3.2 present an asymmetric single line emission feature in their spectra that cannot be attributed to the QSOs emission lines, possibly indicating a high-redshift LAE lensed by the QSO. In Sect. 4 we discuss the completeness of our algorithm search for quasar lenses. We then review the number of QSO-ELG candidates obtained compared to Courbin et al. (2010).

\section{Mining BOSS for candidate QSO lenses}

The SDSS-III BOSS survey provides an unparalleled sample of 297301 QSO optical spectra, with wavelength coverage $3600 \AA-10400 \AA$ and resolution $R \approx 1500-2000$, all inspected and confirmed by eye in Pâris et al. (2017) for Data Release 12. This datasets yields a huge potential for the discovery of QSOs acting as strong lenses. In this section, we detail our search technique.

The selection method used for our sample is based on the one used for the BELLS, SLACS, S4TM and BELLS GALLERY surveys, but with significant changes to select Lyman- $\alpha$ or nebular emission lines superimposed on QSO spectra. The basic principle is to identify additional emission lines distinct from the expected spectral features of the foreground QSO. In this study, we first searched for QSO-Emission Line Galaxy (ELG) systems by looking for additional $\mathrm{OII}, \mathrm{H} \beta$, OIII and/or $\mathrm{H} \alpha$ lines, limiting the redshift of the source to $z_{\mathrm{s}} \lesssim 0.9$ due to the spectrograph wavelength range. Secondly, we searched for QSO-Lyman- $\alpha$ Emitter (LAE) systems, where the asymmetry of the Lyman- $\alpha$ is a signpost for the observed line, allowing us to derive the redshift of the source even though only one line is detected. Detecting Lyman- $\alpha$ emission from a background source in SDSS spectra implies $2 \lesssim z_{\mathrm{S}} \lesssim 6.8$. The lower limit for any background source redshift is also constrained by the foreground QSO redshift.

Detecting QSOs (rather than galaxies) acting as strong lenses via the detection of additional lines is difficult for several reasons. The SDSS pipeline fits the QSOs with a template constructed from the first five eigenvectors of principal component analysis (PCA) decomposition performed on a chosen set of SDSS DR7 QSOs (Pâris et al. 2011), which provides correctly estimated redshifts for most of DR12 QSOs. However, the PCA template is far from perfect and is known to have serious limitations when it comes to reproducing strong broad emission lines or less frequent lines in QSOs spectra (see Fig. 1). Even though we can take advantage of the PCA to approximate the QSO spectra at first order, a range of features are bound to missed by the PCA and might be mistaken for background features. By contrast, the spectral templates of galaxies perform well for SDSS galaxies for that purpose (e.g. Bolton et al. 2006; Brownstein et al. 2012; Shu et al. 2016a). This issue can be overcome by masking broad emission lines where the PCA is most likely to fail, but this limits the search range, and a timeconsuming visual inspection and selection of lens candidates is still necessary to ensure a robust selection. Last but not least, the QSO outshines the lensed background source. This prevents the confirmation of the lensing systems with wide-field photometric data and highlights the crucial importance of high-resolution imaging to confirm such lenses (Courbin et al. 2010, 2012).

\subsection{Preparation of the dataset}

Given the limitations of the PCA for the task at hand, we selected a subset of the BOSS DR12Q (Pâris et al. 2017) dataset to limit future false positive detections. This procedure is summarised in the following steps.

1. We retain only spectra classified as "QSO" by the SDSS pipeline and remove any QSO found in the blank sky fibers. We discarded any QSO fitted by the pipeline with flags ZWARNING! = 0, Z_ERR $<0$ or a reduced $\chi_{\text {fit }}^{2}>10$, which signal potentially wrong redshifts or poor continuum fitting. We also dropped any QSO that has a PCA redshift differing by $>\Delta z>0.1$ from its visually determined redshift.

2. The best-fitted QSO PCA template spectrum provided by the BOSS pipeline was subtracted from the spectra.

3. We masked the main QSO emission lines by setting the inverse variance of the spectra to 0 , preventing any detection of residual QSO emission lines. The same procedure was applied to sky lines to prevent any spurious detection. The masked emission lines are presented in Tables B.1 and B.2 as well as graphically in the upper panel of Fig. 1. The masked fraction of the observed spectra is typically about $\sim 25 \%$. Nonetheless, this masked fraction increases with redshift for multiple reasons. First of all, emission lines cannot be searched in the Lyman- $\alpha$ forest of the quasar, reducing dramatically the search space at $z \gtrsim 2$. Secondly, rest-frame QSO UV broad lines (e.g. N v,Si IV, C IV, and $\mathrm{Mg}$ II) are typically broader than rest-frame optical lines. Finally, the observed width of all lines increases with redshift. We detail in Sect. 4.1 the impact of the masking on the completeness of the lens search.

\subsection{First emission feature search}

After subtracting the PCA template from every suitable QSO spectra selected above, we search for high signal-to-noise ratio 


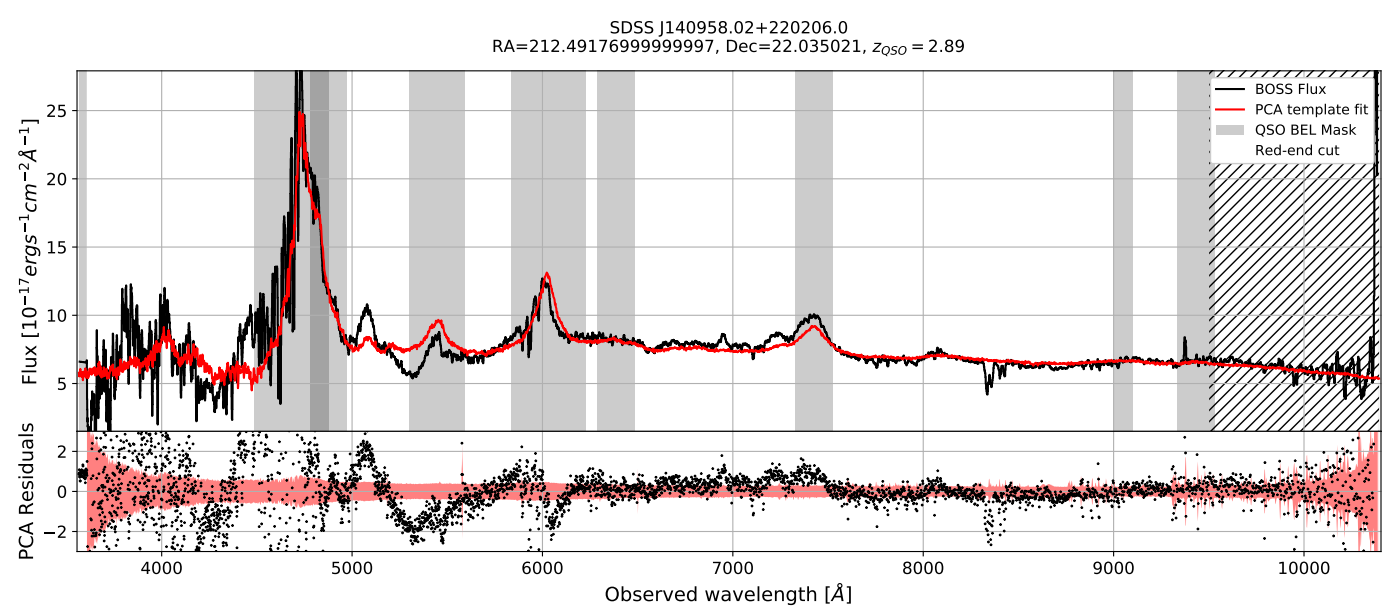

Fig. 1. Typical example of a large misfit between the SDSS pipeline PCA template and the QSO spectra. The grey shaded regions are the masked QSO emission line regions of Table B.1 and sky emission lines of Table B.2. The measured flux is in black, the pipeline PCA template spectrum in red. The lower panel shows the residuals from the PCA template fit subtraction in black and the SDSS spectrograph $1 \sigma$ error array in red. The large residuals, even outside masked regions, create many false positives when searching for extra emission lines from a different object in the QSO spectrum.
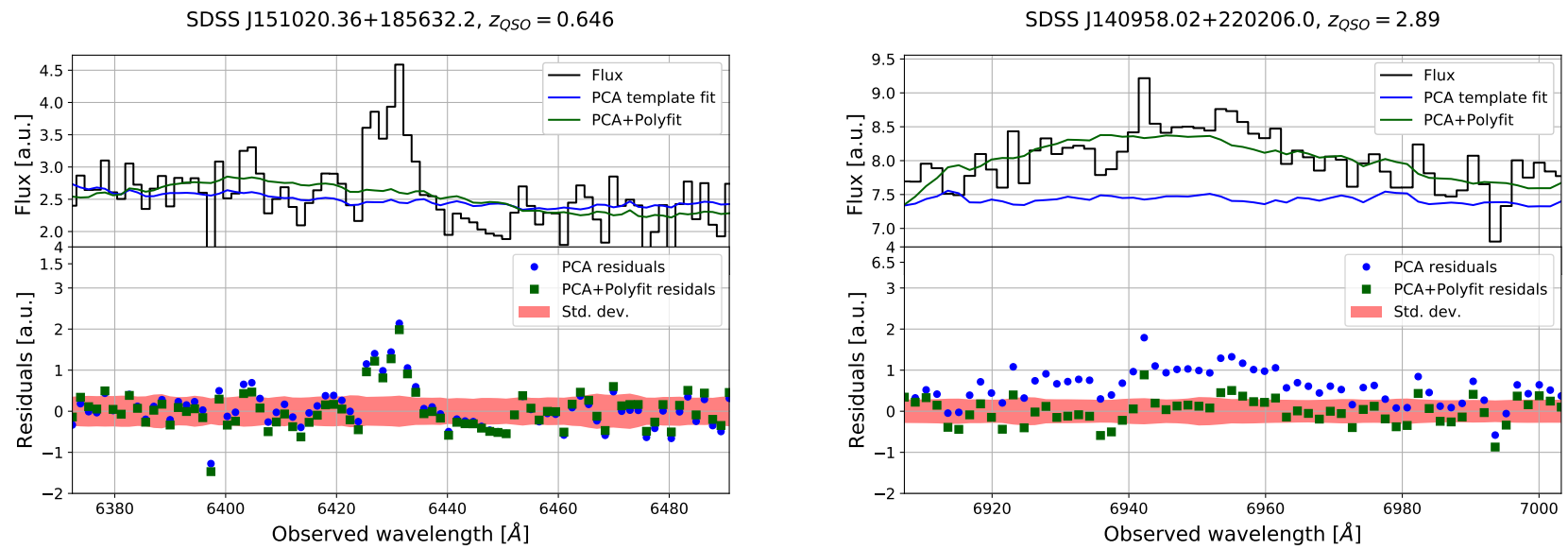

Fig. 2. Comparison between a true emission line detection (right) and a false detection generated by an incorrect PCA template fit (left). However, locally fitting (and subtracting) a third order polynomial to a $40 \AA$ section of the spectra around the line candidate is sufficient to remove continuum features unsubtracted by the PCA. The flux is in black, the PCA template in blue, and the polynomial fit in green. The lower panel showcases the SDSS spectrograph $1 \sigma$ error array in red, and the residuals from both fits in the same colour code. The residuals of the PCA fit are removed and no longer result in a detection, whereas the thin emission line is still clearly detected.

$(\mathrm{S} / \mathrm{N})$ features in the residuals. In order to do this, we made use of a simple matched-filtering approach which is a key step of all spectroscopic selection methods used in the SLACS, BELLS, S4TM, and BELLS GALLERY strong lensing samples and was first presented in Sect. 3.1 of Bolton et al. (2004). We reproduce it here for self-consistency.

The matched-filter search done by convolving a Gaussian kernel $\left\{u_{i}\right\}$ with the spectrum residuals $\tilde{f}_{i}$. The maximum likelihood estimator of the line flux at pixel $j$ with amplitude $A_{j}$ is the one minimising the $\chi^{2}$ value

$\chi_{j}^{2}=\sum_{i} \frac{\left(A_{j} u_{i}-\tilde{f}_{(j+i)}\right)^{2}}{\sigma_{(j+i)}^{2}}$,

where $\tilde{f}_{i}$ is the reduced flux at bin $i$ and $\sigma_{i}$ the measured variance. Setting the derivative by $A_{j}$ to 0 gives the maximum likelihood estimator

$\bar{A}_{j}=C_{j}^{(1)} / C_{j}^{(2)}$, where the two coefficients $C_{j}^{(1)}, C_{j}^{(2)}$ are defined as

$$
\begin{aligned}
& C_{j}^{(1)}=\sum_{i} \frac{\tilde{f}_{j+i} u_{i}}{\sigma_{(j+i)}^{2}}, \\
& C_{j}^{(2)}=\sum_{i} \frac{u_{i}^{2}}{\sigma_{(j+i)}^{2}} .
\end{aligned}
$$

Assuming uncorrelated Gaussian errors on $f_{i}$, the $\mathrm{S} / \mathrm{N}$ of the estimator $\bar{A}_{j}$ is

$$
S / N_{j}=C_{j}^{(1)} / \sqrt{C_{j}^{(2)}} \text {. }
$$

This estimator is quick to compute on all pixels of the residuals, giving an estimate of the $\mathrm{S} / \mathrm{N}$ of potential Gaussian emission features at each pixel. In the following, we refer to this estimator as the $\mathrm{S} / \mathrm{N}$ for simplicity. The matched-filter feature search is performed on all pixels of each spectrum. We used a Gaussian kernel with a dispersion $\sigma=150 \mathrm{~km} \mathrm{~s}^{-1}$ and store detections above $8 \sigma$. 

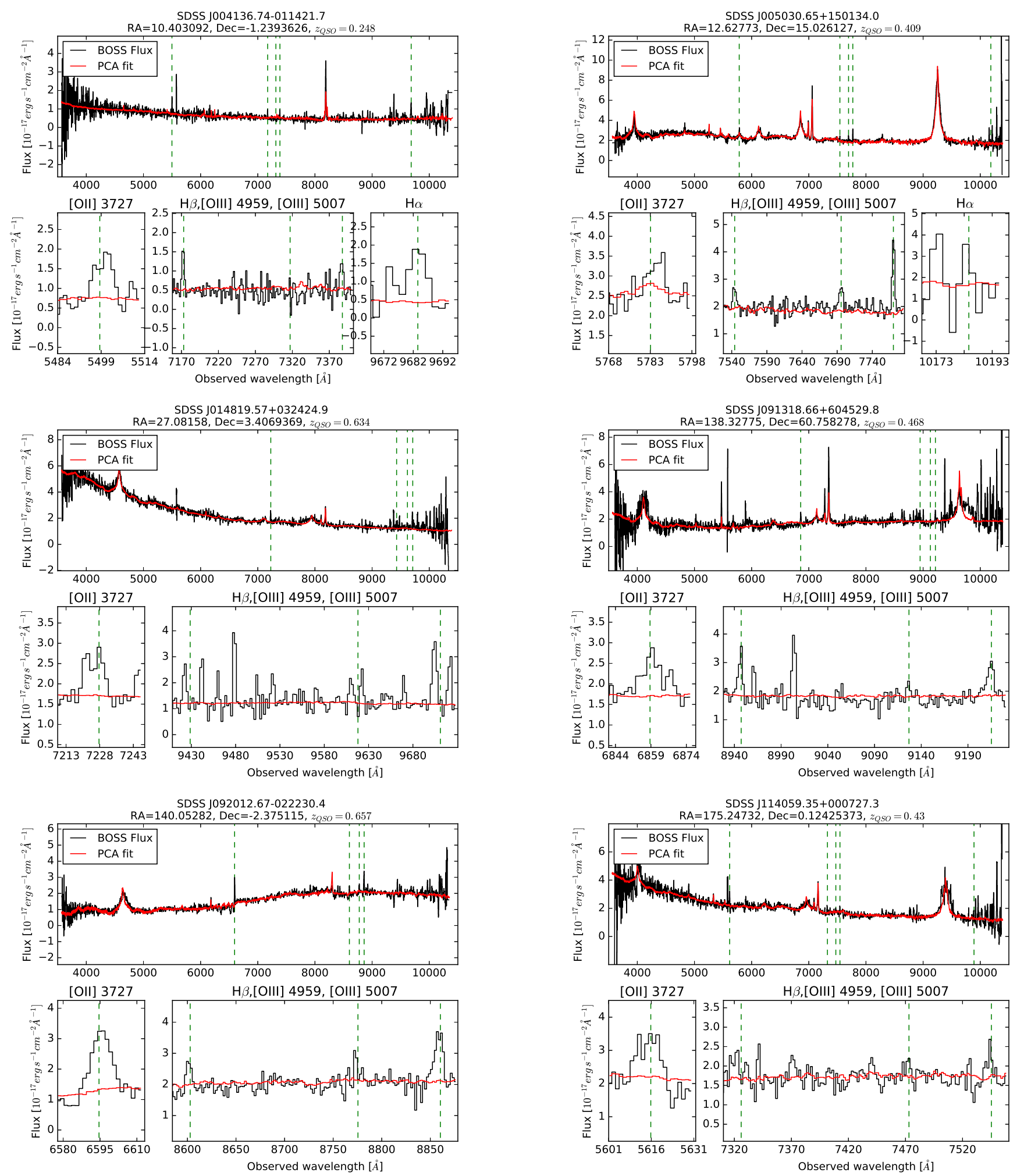

Fig. 3. First six QSO-ELG candidates. Upper panels: five-pixel smoothed BOSS spectra (black) and QSO fitted PCA template (red). The location of the background galaxy emission lines are indicated by dashed green lines. Lower panels: zoom on the detected emission lines of the source galaxies.

\subsection{Algorithmic search for background features}

In this section, we describe our algorithm for selecting secure background objects based on the detections of significant features described above. We first searched for multiple nebular emission lines at a similar redshift present in the QSO spectrum residuals to identify QSO-ELG lens candidates. Then, we sorted the remaining extra single emission lines to select QSO-LAE lens candidates using the distinct asymmetry of the Lyman- $\alpha$ profile. 



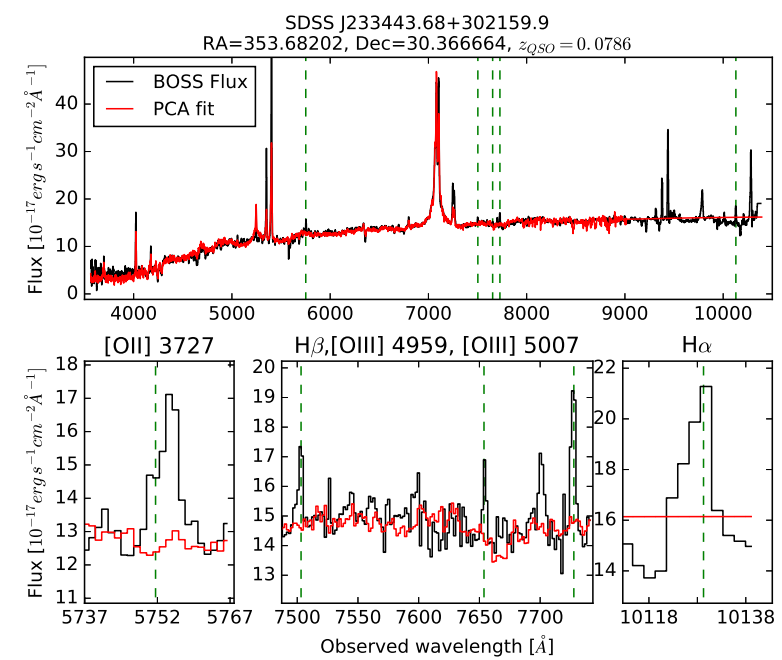

Fig. 4. Spectroscopy of the three last QSO-ELG system candidates. Upper panels: five-pixel smoothed BOSS spectra (black) and QSO fitted PCA template (red). The location of the background galaxy emission lines are indicated by dashed green lines. Lower panels: zoom on the detected emission lines of the source galaxy.

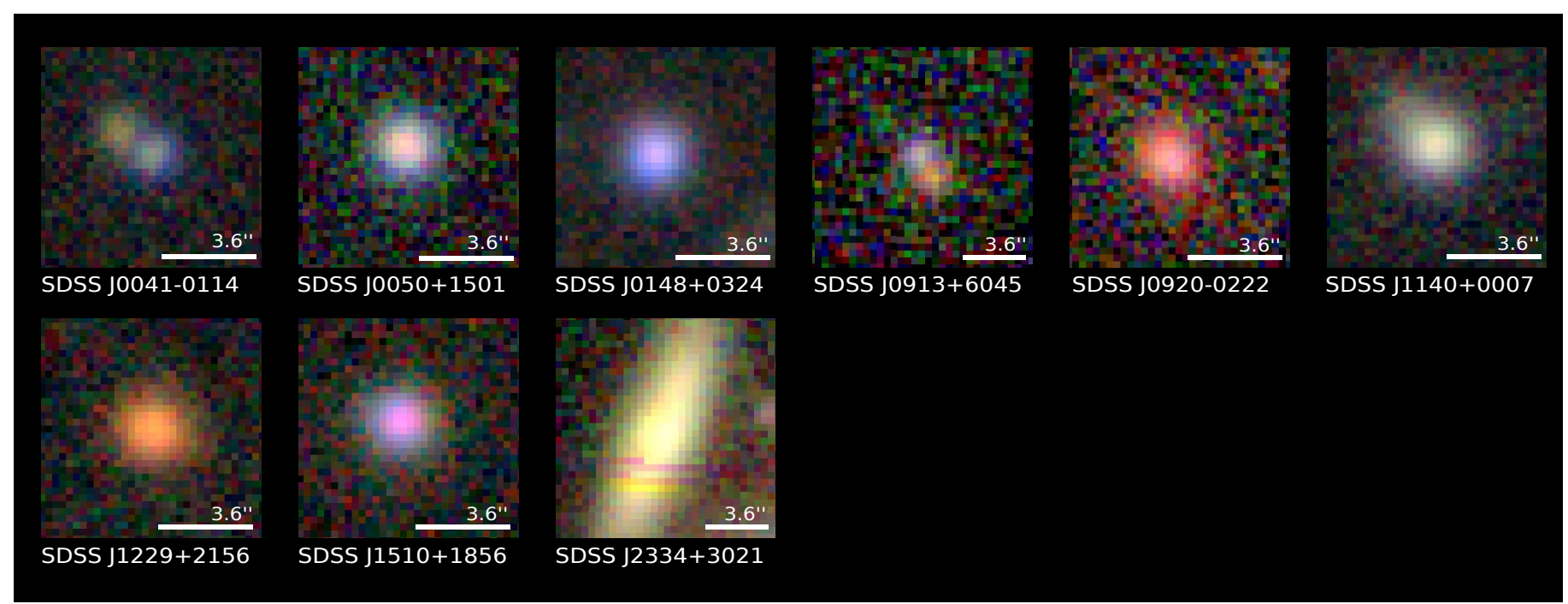

Fig. 5. DELS/SDSS imaging of the nine QSO-ELG candidates. The stamps are centred on the BOSS targets, and the thick white line indicates the diameter of the BOSS fiber $\left(2^{\prime \prime}\right)$ augmented by the mean 80 th percentile seeing $\left(\sim 1.6^{\prime \prime}\right)$. As expected, no candidates prevent evidence of lensed features, but nearly half of them display a nearby redder object. 
Table 1. Selected properties of the nine QSO-ELG lens candidate systems.

\begin{tabular}{|c|c|c|c|c|c|c|c|c|c|c|c|c|}
\hline Target & RA & Dec & Plate-MJD-Fiber & $z_{\mathrm{QSO}}$ & $z_{\mathrm{s}}$ & $S / N_{1}$ & $S / N_{\text {tot }}$ & $g$ & $i$ & $M_{\mathrm{SIS}}$ & $f_{\mathrm{OII}}$ & $f_{\text {OIII }}$ \\
\hline SDSS J004136.7 & & & & O & & & & 21.01 & 6 & 2.9 & 7.43 & 02.6 \\
\hline & & & & & 0.55 & 14.13 & & & & 8.1 & 3.42 & 13.4 \\
\hline & & & & 0.634 & 0.94 & 33 & & & & 8.7 & 8.94 & 18.4 \\
\hline & & & & & 0.84 & 8.36 & & & & 5.2 & 10.61 & $9.7^{(1)}$ \\
\hline & & & & & 0.77 & 14.77 & & & & 19.6 & 16.53 & 15.7 \\
\hline & & 0.10 & & 0.430 & 0.51 & 8.32 & & & & 14.2 & 9.01 & 2.7 \\
\hline & & & & & 0. & & & & & 6.6 & 1 & 09.0 \\
\hline & & & & & 0.7 & 9. & & & 19. & 26.0 & & 23.7 \\
\hline SDSS J233443.68+302159.9 & 353.68202 & 30.366664 & $6501-56563-228$ & 0.079 & 0.54 & 10.33 & 18.17 & 19.33 & 17.69 & 0.6 & 22.37 & 17.6 \\
\hline
\end{tabular}

Notes. Description of the fields: (1) SDSS target name in terms of truncated J2000 RA and Dec in the format HH.MM.SS.ss +DD.MM.SS.s. (2) Right Ascension (RA) in degrees. (3) Declination (Dec) in degrees. (4) Plate-MJD-Fiber of the spectrum. (5) QSO redshift from the BOSS pipeline. (6) Background galaxy redshift inferred from the emission lines detection. (7) $\mathrm{S} / \mathrm{N}$ of the strongest emission line. (8) Quadrature sum $\mathrm{S} / \mathrm{N}$ of the emission lines. (9) $g$-band magnitude. (10) $i$-band magnitude. (11) Mass (10 ${ }^{12} M_{\odot}$ ) enclosed within the maximum detectable Einstein radius $\theta_{\mathrm{E}}=3.6^{\prime \prime}$, assuming a singular isothermal sphere (SIS) model and flat $\Lambda$ CDM cosmology (Planck Collaboration XIII 2016), giving an approximate upper bound on the lens mass. (12) Apparent flux (continuum subtracted) of the [OII] $\lambda 3727 \AA$ emission line in units of $10^{-17} \mathrm{erg} \mathrm{cm}^{-2} \mathrm{~s}^{-1}$. (13) Apparent flux (continuum subtracted) of [OIII] $\lambda 5007 \AA$ emission line in units of $10^{-17} \mathrm{erg} \mathrm{cm}^{-2} \mathrm{~s}^{-1}$. ${ }^{(1)}$ SDSS J0148+0324 does not meet the criteria for QSO-ELG selection, but exhibits doubled emission lines, as visible in Fig. 3, probably due to two background galaxies. The flux reported above corresponds to only one emission line.

Table 2. Selected properties of the QSO-LAE lens candidate systems.

\begin{tabular}{|c|c|c|c|c|c|c|c|c|c|c|c|}
\hline Target & RA & Dec & Plate-MJD-Fiber & $z_{\mathrm{L}}$ & $z_{\mathrm{s}}$ & $S / N_{\mathrm{Ly} \alpha}$ & $a_{\lambda}$ & $g$ & $i$ & $M_{\mathrm{SIS}}$ & $f_{\mathrm{LyA}}$ \\
\hline SDSS J013934.96+010629.9 & 24.895706 & 1.1083212 & $4231-55444-622$ & 0.96 & 4.01 & 9.82 & 2.24 & 22.41 & 21.33 & 4.9 & 8.80 \\
\hline SDSS J101625.37+503427.0 & 154.10572 & 50.574194 & $6668-56605-342$ & 1.23 & 4.48 & 11.24 & 1.93 & 20.23 & 19.67 & 5.8 & 11.13 \\
\hline SDSS J235422.48+195141.3 & 358.59368 & 19.861487 & $6110-56279-406$ & 0.75 & 2.17 & 10.66 & 0.21 & 18.64 & 18.40 & 4.9 & 72.31 \\
\hline
\end{tabular}

Notes. Description of the fields: (1) SDSS target name in terms of truncated J2000 RA and Dec in the format HH.MM.SS.ss+DD.MM.SS.s. (2) Right Ascension (RA) in degrees. (3) Declination (Dec) in degrees. (4) Plate-MJD-Fiber of the spectrum for the BOSS target. (5) QSO redshift from the BOSS pipeline. (6) Background LAE redshift inferred from the peak detection. (7) Signal-to-noise ratio of the detected line. (8) Wavelength ratio $a_{\lambda}$ (Rhoads et al. 2003), further described in Sect. 3.2. (9) $g$-band magnitude. (10) $i$-band magnitude. (11) Mass (10 ${ }^{12} M_{\odot}$ ) enclosed within the maximum detectable Einstein radius $\theta_{\mathrm{E}}=3.6^{\prime \prime}$, assuming a singular isothermal sphere (SIS) model and flat $\Lambda \mathrm{CDM}$ cosmology (Planck Collaboration XIII 2016), giving an approximate upper bound on the lens mass. (12) Apparent flux (continuum subtracted) of the Lyman- $\alpha$ emission in units of $10^{-17} \mathrm{erg} \mathrm{cm}^{-2} \mathrm{~s}^{-1}$.

1. We limited this search to the region $\left(1+z_{\mathrm{QSO}}\right) \lambda_{\mathrm{Lyman}-\alpha}<$ $\lambda_{\text {observed }}<9500 \AA$ to avoid the Lyman- $\alpha$ forest and the red edge of the SDSS spectrograph, highly populated by sky emission lines. All matched-filtered detections outside this range are immediately discarded.

2. A third order polynomial is fitted in a $\sim 6000 \mathrm{~km} \mathrm{~s}^{-1}$ velocity range centred on detections to remove any contribution from a poor PCA continuum fit. The $\mathrm{S} / \mathrm{N}$ is recomputed and we keep only features with a new $\mathrm{S} / \mathrm{N}$ above $6 \sigma$ to account for the fact that the polynomial might cause a small S/N loss to true positives. Large un-subtracted continuum residuals are discarded at this step, as the $\mathrm{S} / \mathrm{N}$ would otherwise drop well below the required $6 \sigma$. Figure 2 illustrates this process on both a typical true- and false-positive detections.

3. For each QSO, the detection candidates are ranked by $\mathrm{S} / \mathrm{N}$ and only the five highest are kept. Multiple background sources in the $2^{\prime \prime}$ SDSS fiber are extremely unlikely to be detected. In practice, quasars with more than five lens candidates have an extremely poor continuum fit or present a lot of noise in their spectra. If all background optical emission lines ([OII], $\mathrm{H} \beta$, [OIII], and $\mathrm{H} \alpha$ ) were strong enough to be detected with the gaussian-matched filter, we would expect at most five detections. We thus limited the number of candidates to five to reduce the number of candidates that will need to be visually inspected.

4. We first checked whether each detection might be part of a larger group of lines at a higher redshift than the QSO as it is unambiguous signal for an aligned ELG. We ascribed each peak

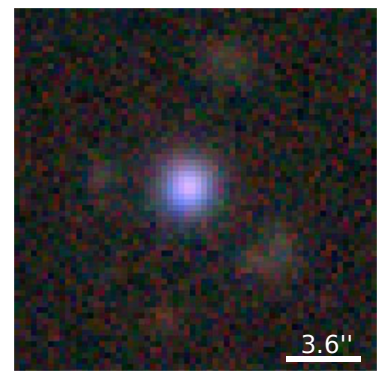

Fig. 6. Enlarged view of QSO-ELG candidate J0148+0324. The DELS composite shows four similar faint images in an Einstein cross configuration, albeit at a somewhat large image separation. It is the only candidate featuring such intriguing hints of strong lensing. Surprisingly, this candidate is also remarkable by the presence of not one, but two series of detected background emission lines at a very close velocity separation (see Fig. 3).

in turn to OII, $\mathrm{H} \beta$, OIII, and $\mathrm{H} \alpha$ at $z_{\mathrm{ELG}} \neq z_{\mathrm{QSO}}$. We then looked up the $\mathrm{S} / \mathrm{N}$ at the expected position of the four other redshifted emission lines. If the quadrature sum $\mathrm{S} / \mathrm{N}$ of the pixels at the expected line positions is greater than initial detection $\mathrm{S} / \mathrm{N}$ by at least $2.5 \sigma$ (for a total $\mathrm{S} / \mathrm{N}$ of $10.5 \sigma$ ), we flagged this candidate as a "QSO-ELG" lens candidate. A total of 254 "QSO-ELG" objects were flagged.

5. This leaves 929 detections that cannot be safely attributed to an ELG and were hence dubbed "QSO-Single Line Emitter" 
SDSS J013934.96+010629.9

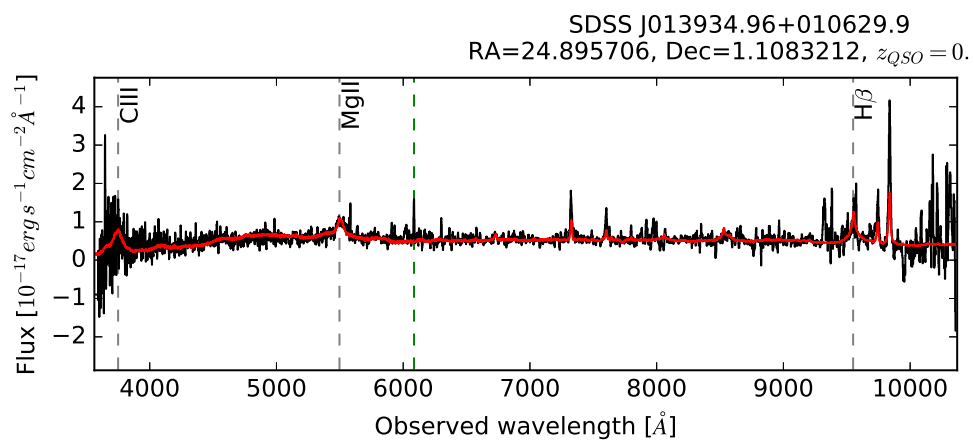

SDSS J101625.37+503427.0

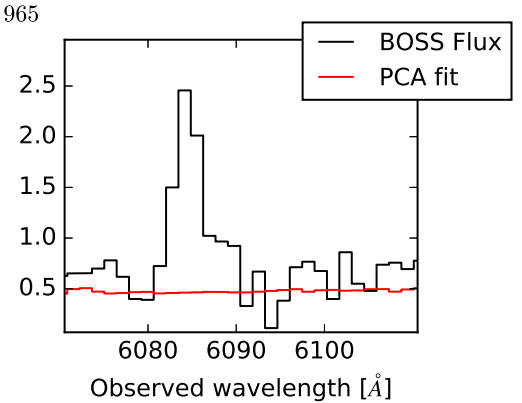

$\mathrm{RA}=154.10572, \mathrm{Dec}=50.574194, z_{Q S O}=1.23$
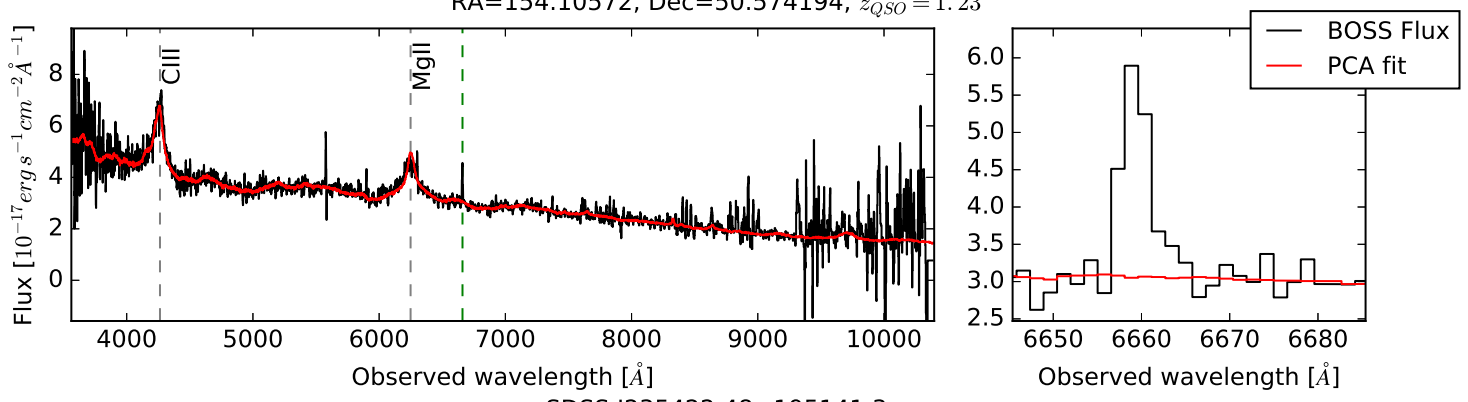

SDSS J235422.48+195141.3
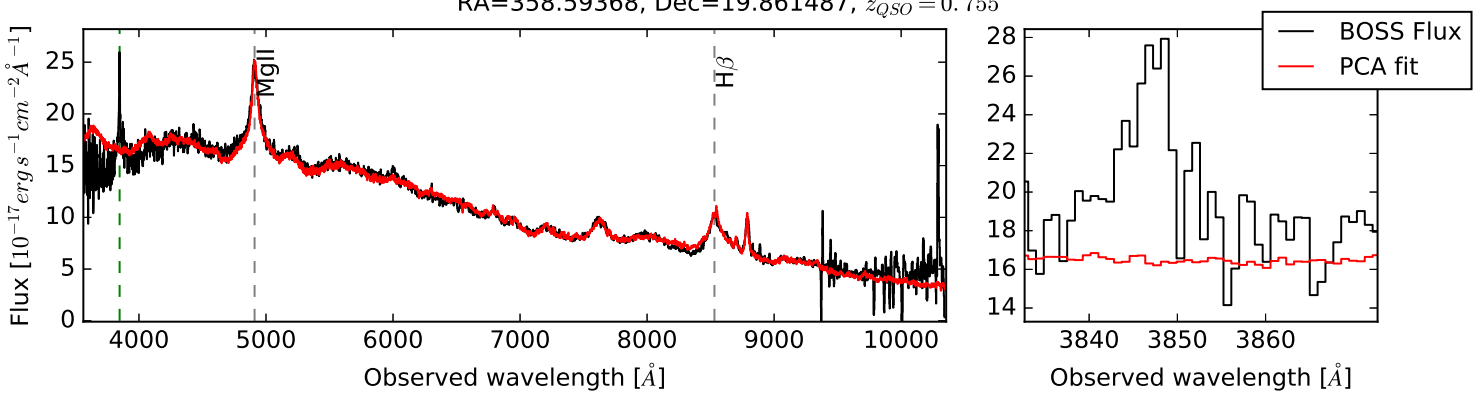

Fig. 7. SDSS spectra of the QSO-LAE lensing systems candidates. Right panels: 5-pixel smoothed BOSS spectra (black) and QSO fitted PCA template (red). The detected extra emission line is indicated by the dashed green line. Left panels: zoom on the original spectra of the candidate lensed Lyman- $\alpha$ emission line. We note the asymmetric profile characteristic of Lyman- $\alpha$.

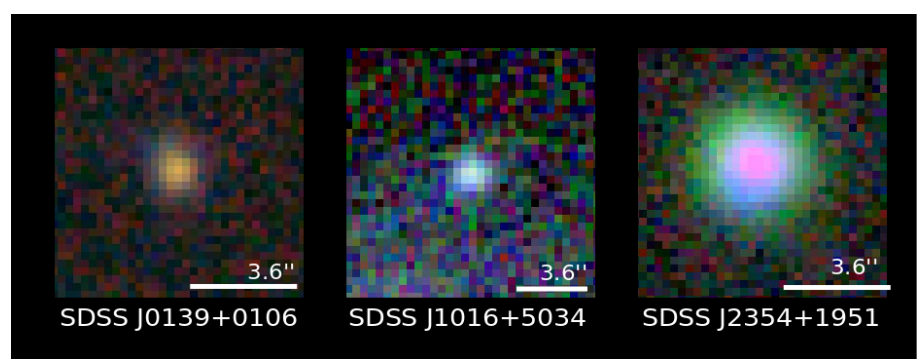

Fig. 8. DELS/SDSS imaging of the three QSO-LAE candidates. The stamps are centred on the BOSS targets, and the thick white line indicates the diameter of the BOSS fiber $\left(2^{\prime \prime}\right)$ augmented by the mean 80 th percentile seeing $\left(\sim 1.6^{\prime \prime}\right)$. As expected, no candidates prevent evidence of lensed features.

system (QSO-SLE). They were subsequently visually inspected for asymmetry in the detected line as a hint for Lyman- $\alpha$ emission.

Eventually all QSO-ELG and QSO-LAE lens candidates are inspected by eye and their SDSS or Dark Energy Spectroscopic Instrument (DESI) Legacy Imaging Surveys (DELS; Dey et al. 2019) imaging was checked to remove any hits possibly due to nearby galaxies. The nearby fibers were also inspected to check for any strong line at the detected wavelength to avoid any false positive due to cross-talk between neighbouring fibers. This leaves a total of nine secure QSO-ELG candidates presented in Table 1. Most of the removed algorithmic candidates were obvious failures of the template and polynomial fits, or unconvincing lines in noisy parts of the spectra. Through visual inspection, we also selected three potential QSO-LAE candidates presented in Table 2. We also record 49 single line emitter (SLE) detections that were not discarded during visual inspection but were not deemed sufficently asymetric to be attributed to a background LAE (see Appendix A). The SLEs redshift distribution is presented in Fig. 9 alongside the QSO-ELG and QSO-LAE candidates source and lens redshift distributions.

\section{Lens candidates samples}

\subsection{QSO-ELG candidates}

The nine QSO-ELG candidates were selected to present clear visual evidence of extra background emission lines. All of them are selected using the above $8 \sigma$ detection threshold of an initial line (usually OII or $\mathrm{H} \beta$ ), along with evidence for other lines by requiring a quadrature sum $\mathrm{S} / \mathrm{N}$ of all lines $>10.5 \sigma$. In practice, all but one have a total $S / N>10.5 \sigma$. The nine candidates all present four or five significant emission lines among [OII] 3727, H $\beta$, [OIII] 4959, [OIII] 5007 and $\mathrm{H} \alpha$ (see 


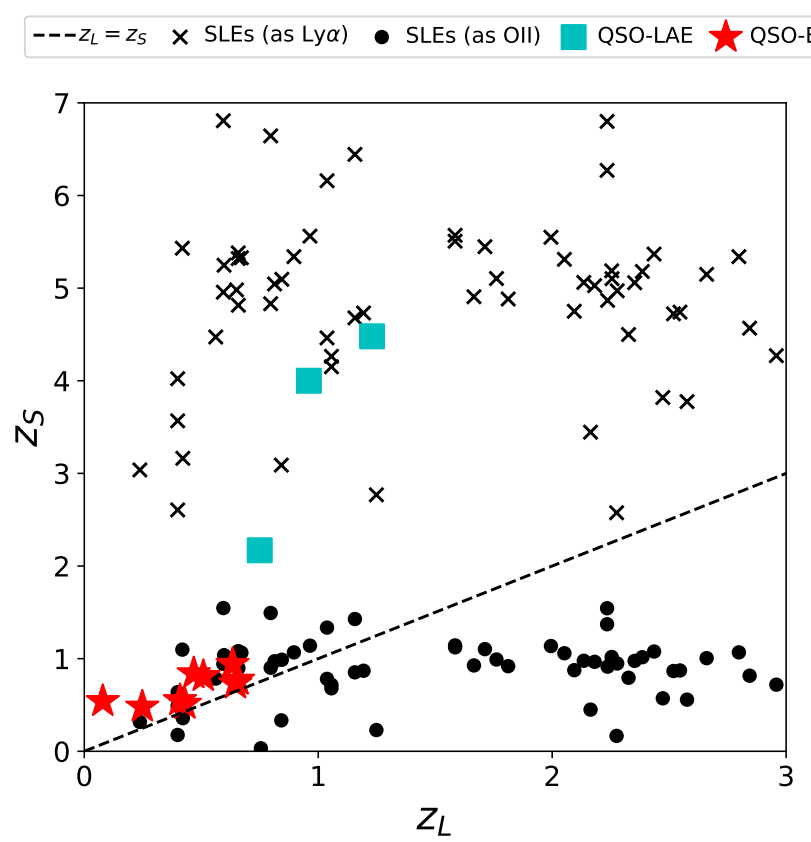

Fig. 9. Lens $\left(z_{\mathrm{L}}\right)$ and source $\left(z_{\mathrm{S}}\right)$ redshift distributions of the 9 QSOELG lens candidates, the 3 QSO-LAE lens candidates and the 49 remaining SLEs selected by the algorithm that were not shortlisted as QSO-LAE candidates (see Appendix A for some examples), with redshift are determined assuming either background Lyman- $\alpha$ or [O II] emission. In the case of QSO-LAEs, our selection method seems to favour low redshift QSOs and high-redshift background sources. QSOELG candidates however have both low lens and source redshifts. The lower and upper boundaries on the source redshift are due to the limited range of the SDSS spectrograph. The dotted line indicates the $z_{\mathrm{S}}>z_{\mathrm{L}}$ limit for background object detections.

Figs. 3 and 4). Selected properties of the QSO-ELG lens candidate sample are presented in Table 1, and the source and lens redshift distributions of the systems are presented in Fig. 9. Three candidates (SDSS J1140+0007, SDSS J0041+0114 and SDSS J0913+6045) present either nearby features surrounding the QSO or a distinct nearby object in the DELS or SDSS colour composite images shown in Fig. 5.

$\mathrm{J} 0148+0324$ presents four distinct features disposed around it in a seemingly Einstein Cross configuration (see Fig. 6). However, we note that the Einstein radius $\sim 4^{\prime \prime}$ would be quite large for such a strong lensing system. Coincidentally or not, J0148 also presents two close series background emission lines (Fig. 3) at $\sim 400 \mathrm{~km} \mathrm{~s}^{-1}$ separation. We hypothesise that these intriguing characteristics could be due to a small background cluster or group lensed by the quasar.

\subsection{QSO-LAE candidate lenses}

Our three QSO-LAE candidates were visually selected from the 49 SLE sample to present asymmetric Lyman- $\alpha$ profiles. We exclude low-redshift OII emission by checking for the absence of emission at the expected redshifted $\mathrm{H} \beta$, OIII, $\mathrm{H \alpha}$ wavelength. The QSO-LAE candidates redshift distribution is presented in Fig. 9 along with the other SLEs under the assumption that the single line is either Lyman- $\alpha$ or [OII]. The spectroscopy of the 3 QSO-LAE is shown in Fig. 7. The SDSS or DELS imaging of each QSO-LAE is presented in Fig. 8 but does not confirm the presence of strong gravitational lensing features. As in Courbin et al. (2012), we do expect the lensed images to be outshined by the QSO in the SDSS photometry and thus to be revealed only by high-resolution imaging.

\section{Discussion}

\subsection{Selection function of our algorithm}

Here we assess the selection function of our algorithm, which is of crucial importance to any cosmological applications relying on the number density of quasars acting as lenses. We noted above that the search is limited by the masking of the broad emission lines that are usually badly fitted by the PCA in the SDSS pipeline. The masked fraction of the observed wavelength evolves from $\sim 20 \%$ to $\sim 50 \%$ with redshift as the broader QSO UV lines enter the SDSS spectrum. Additionally, additional emission lines are virtually impossible to detect in the Lyman- $\alpha$ forest of the QSO, reducing even more the wavelength search range for QSO at $z \gtrsim 2$. This severely limits the detection of higher-redshift lensed systems. As we show below (see Figs. 10 and 11), the masking impacts more the detections of background LAEs than ELGs. The first reason is of course that ELGs are searched for at $z \lesssim 1$, where the masking is only about $\lesssim 20 \%$ of the observed wavelength range. The second is that ELGs can rely on five different lines distributed throughout the spectra, and so the masking of one does not prevent a successful detection.

We computed the completeness of our search by inserting mock ELG emission lines (only [OII], [OIII], $\mathrm{H} \beta$ and $\mathrm{H} \alpha$ at the same $\mathrm{S} / \mathrm{N}$ for simplicity), and mock SLEs in random SDSS spectra. We modelled the emission lines by Gaussian profiles with a variance of 1.2 pixels, rescaled to match a chosen $\mathrm{S} / \mathrm{N}$. We divided the $\left(z_{\mathrm{L}}, z_{\mathrm{S}}\right)$ space in a fine grid for which we insert 1000 emission features in randomly selected foreground QSOs spectra from $\mathrm{DR} 12 \mathrm{Q}$. The process is repeated for all $\mathrm{S} / \mathrm{N}$ in the range $[4,12]$. We present in Figs. 10 and 11 the completeness of our algorithmic search for ELGs and SLEs, respectively. We first note the overall good performance of the search for ELGs, and its good completeness at $S / N=8$ which was the threshold chosen for feature detection for this study. As said above, the ELG search is only lightly impeded by the broad line masking because it can use up to five different lines to detect a background galaxy. The masking of QSO emission lines creates linear traces of low-completeness in the $\left(z_{\mathrm{S}}, z_{\mathrm{L}}\right)$ space. However, this affects relatively more systems with with $z_{\mathrm{L}}>0.7$. Indeed, at $z_{\mathrm{L}}>0.7$ [OIII] moves out of the allowed wavelength range $(<8500 \AA)$ and thus candidates are retrieved only if both [OII] and $\mathrm{H} \beta$ are detected. If one of them falls close to one of the broad QSO emission lines, the completeness drops close to zero. At $z<0.7$, this effect is mitigated since more than three lines can be detected and the probability of them all falling into masked regions of the spectra is virtually null.

The SLE completeness is about a factor 30-50\% lower compared to ELG at the same S/N, which cannot only be attributed to a larger sensitivity to the masking. Indeed, all ELG features are masked only for specific combinations of $\left(z_{\mathrm{S}}, z_{\mathrm{L}}\right)$, whereas single emitter lines are never detected if they fall in some of the masked regions, which can make up a large fraction of the spectra. The remaining drop in efficiency is easily explained by the scatter in the estimated $\mathrm{S} / \mathrm{N}$ and the real $\mathrm{S} / \mathrm{N}$. When multiple lines are used, the probability that all of them are underestimated due to continuum residuals is low, whereas an underestimated SLE close to the $\mathrm{S} / \mathrm{N}$ threshold is always rejected.

The purity of the algorithmic search requires a comprehensive modelling of QSO spectra and background sources and is thus beyond the scope of this paper. The human confirmation process of course tends to increase purity and diminish completeness, 



Fig. 10. Completeness function for the QSO-ELG lens system search with a detection threshold set at $S / N=4,6,8,10$ (left to right). The completeness rises quickly to 1 above the set threshold and is only slightly impeded by the masking of broad and narrow QSO emission lines, as visible in linear trends of low completeness in the $\left(z_{\mathrm{S}}, z_{\mathrm{L}}\right)$ plot.

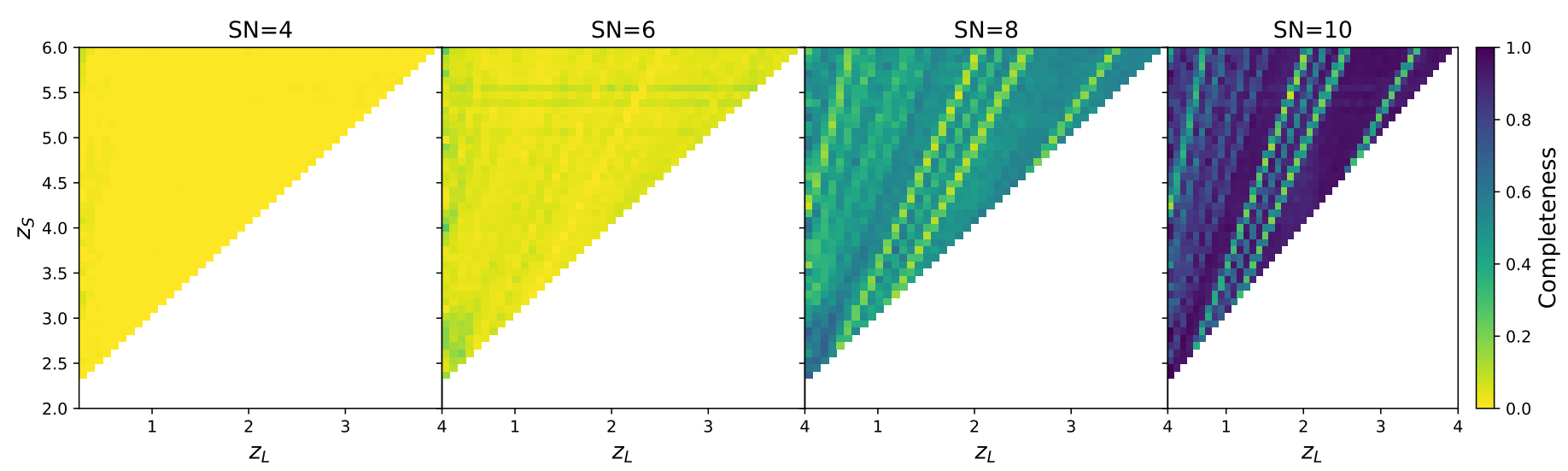

Fig. 11. Completeness function for the QSO-SLE lens systems search with a detection threshold set at $S / N=4,6,8,10$ (left to right). The completeness is heavily impacted by the masking of broad and narrow QSO emission lines, as visible in linear trends of low completeness in the $\left(z_{\mathrm{S}}, z_{\mathrm{L}}\right)$ plot.

but we are confident that this process is negligible for the QSOELG lenses sample. Only a small number of systems $(\sim 250)$ had to be checked by eye for ELG features, and the nature of false positives was always obvious (e.g. clear continuum residuals or clipped spectrum). The selection of QSO-LAE lenses is more difficult because measuring the asymmetry of the profile is difficult at the resolution of the BOSS spectrograph where low-S/N features are not well-resolved. We have attempted to classify SLEs based on different asymmetry indicators such as skewness and the Rhoads et al. (2003) wavelength ratio, but they fail to clearly separate candidates selected by eye from the parent SLE sample. We note that no QSO-LAE lens system has been observed to date, thus a first detection would motivate a fully automated selection from spectrosocopic surveys of such lensing systems.

We have hence characterised our selection function, showing that the search for background ELG is complete at the $>90 \%$ for the chosen $\mathrm{S} / \mathrm{N}$ threshold, and that the QSO-LAE search is mostly impeded by the masking of broad emission lines. In the latter case, a more careful modelling of QSO continua could boost the number of detections by a factor of approximately two, which could be very significant given the prospect of future large spectroscopic samples of QSOs.

\subsection{Number of QSO-ELG systems}

We now discuss the number of candidates obtained through our search across all SDSS-III QSOs, and compare it to the only pre- vious search for such lenses. We first draw some parallels and differences between Courbin et al. (2012) first three confirmed QSO-galaxy lenses and our nine candidates. The two candidates samples do not overlap because we did not apply our algorithm to SDSS-I/-II.Indeed, our automated method is built around the PCA template for quasars which was introduced in SDSS-III. We note that all the QSO-ELG lenses shortlisted in Table 1 have a lens redshift $z<0.7$, as did the SDSS-II QSOs in Courbin et al. (2010). Our candidate selection is based on the detection of multiple emission lines among [OII], $\mathrm{H} \beta$, [OIII] and $\mathrm{H} \alpha$. Even though $\mathrm{H} \alpha$ is not essential for a detection, [OII], [OIII] and $\mathrm{H} \beta$ should be clearly visible. This sets a detection limit for background galaxies lensed by QSOs, i.e. $z \lesssim 0.8$, as [OIII] and $\mathrm{H} \beta$ at higher redshift are off the red end of the SDSS spectrograph. As the red end of the BOSS spectrograph is often too noisy and affected by strong sky emission, only $z \lesssim 0.7$ QSOs can in fact be used to detect QSOELGs systems. BOSS primarily targeted QSOs at redshift $z>2$. Hence we are limited by the number of QSOs in the low-redshift tail of the distribution of BOSS QSOs, or objects re-observed from SDSS-I/II. The number of QSOs with redshift $z<0.7$ is 31081 in BOSS DR12 and thus we reach a fraction of selected QSO-ELG lens candidates of $9 / 31081 \approx 3 \times 10^{-4}$.

\subsection{Future perspectives}

We demonstrate the potential of our spectroscopic selection of QSO lensing background ELGs/LAEs where image-based 
techniques often fail due to the brightness of the foreground target. In the case of QSO-ELG lenses, the presence of at least four emission lines at a total $\mathrm{S} / \mathrm{N}$ of $\gtrsim 11 \sigma$ guarantees the presence of a higher-redshift background galaxy. With high spatial resolution follow-up, this sample may increase the number of such QSO lenses by a factor of three to four.

QSO-LAE lens candidates are more tentative candidates. However, there is hope to confirm the first ever QSO-LAE strong lensing system. The remaining SLEs sample could contain more QSO-LAE candidates, but there is not enough evidence to draw firm conclusions only based on the spectra. We note, however, that selecting galaxies on Lyman- $\alpha$ only was succussful for the BELLS-GALLERY survey. Because Lyman- $\alpha$ can be detected in optical spectra at a wider range of redshifts than nebular emission lines, most QSO acting strong lenses could be in the future QSO-LAE systems. Improving the modelling of the QSO continuum will reduce the masked fraction of the observed spectra, potentially doubling the wavelength search space and thus the number of candidates. Confirming the first QSO-LAE system is thus the next step in the direction of statistically meaningful sample of QSO acting as strong gravitational lenses. This is of prime importance for the applications of large samples: constraining the $M_{\mathrm{BH}}-\sigma_{*}$ relationship from the QSO broadened lines and the mass inferred from the lens modelling, as well as using the observed lensing probabilities to differentiate halo models of QSOs.

Acknowledgements. JPK and TD acknowledge support from the ERC advanced grant LIDA. FC acknowledges support from the Swiss National Science Foundation (SNSF). We thank an anonymous referee for comments on a first version of this paper which significantly improved it. We thank Mathilde Jauzac for useful feedback on an early draft of this manuscript. We are grateful to the authors of the following Python packages: Matplotlib (Hunter 2007), Numpy (van der Walt et al. 2011) and Scipy (Jones et al. 2001). Funding for SDSS-III has been provided by the Alfred P. Sloan Foundation, the Participating Institutions, the National Science Foundation, and the U.S. Department of Energy Office of Science. The SDSS-III web site is http://www.sdss3.org/. SDSSIII is managed by the Astrophysical Research Consortium for the Participating Institutions of the SDSS-III Collaboration including the University of Arizona, the Brazilian Participation Group, Brookhaven National Laboratory, Carnegie Mellon University, University of Florida, the French Participation Group, the German Participation Group, Harvard University, the Instituto de Astrofisica de Canarias, the Michigan State/Notre Dame/JINA Participation Group, Johns Hopkins University, Lawrence Berkeley National Laboratory, Max Planck Institute for Astrophysics, Max Planck Institute for Extraterrestrial Physics, New Mexico State University, New York University, Ohio State University, Pennsylvania State University, University of Portsmouth, Princeton University, the Spanish Participation Group, University of Tokyo, University of Utah, Vanderbilt University, University of Virginia, University of Washington, and Yale University.

\section{References}

Agnello, A., Grillo, C., Jones, T., et al. 2018, MNRAS, 474, 3391 Alam, S., Albareti, F. D., Prieto, C. A., et al. 2015, ApJS, 219, 12 Auger, M. W., Treu, T., Bolton, A. S., et al. 2009, ApJ, 705, 1099 Bolton, A. S., Burles, S., Schlegel, D. J., Eisenstein, D. J., \& Brinkmann, J. 2004, AJ, 127,1860
Bolton, A. S., Burles, S., Koopmans, L. V. E., Treu, T., \& Moustakas, L. A. 2006 ApJ, 638, 703

Bolton, A. S., Burles, S., Koopmans, L. V. E., et al. 2008, ApJ, 682, 964 Bolton, A. S., Brownstein, J. R., Kochanek, C. S., et al. 2012, ApJ, 757, 82 Browne, I. W. A., Wilkinson, P. N., Jackson, N. J. F., et al. 2003, MNRAS, 341, 13

Brownstein, J. R., Bolton, A. S., Schlegel, D. J., et al. 2012, ApJ, 744, 41

Cabanac, R. A., Alard, C., Dantel-Fort, M., et al. 2007, A\&A, 461, 813

Cen, R., \& Safarzadeh, M. 2015, ApJ, 798, L38

Cen, R., \& Safarzadeh, M. 2017, MNRAS, 467, L26

Cornachione, M., Bolton, A., Shu, Y., et al. 2018, ApJ, 853, 148

Courbin, F., Tewes, M., Djorgovski, S. G., et al. 2010, A\&A, 516, L12

Courbin, F., Faure, C., Djorgovski, S. G., et al. 2012, A\&A, 540, A36

Dawson, K. S., Schlegel, D. J., Ahn, C. P., et al. 2013, AJ, 145, 10

Dey, A., Schlegel, D. J., Lang, D., et al. 2019, AJ, 157, 168

Eisenstein, D. J., Weinberg, D. H., Agol, E., et al. 2011, AJ, 142, 72

Faure, C., Kneib, J.-P., Covone, G., et al. 2008, ApJS, 176, 19

Huchra, J., Gorenstein, M., Kent, S., et al. 1985, AJ, 90, 691

Hunter, J. D. 2007, Comput. Sci. Eng., 9, 90

Inada, N., Oguri, M., Shin, M.-S., et al. 2012, ApJ, 143, 119

Jones, E., Oliphant, T., Peterson, P., et al. 2001, SciPy: Open Source Scientific Tools for Python

Joseph, R., Courbin, F., Metcalf, R. B., et al. 2014, A\&A, 566, A63

Kaspi, S., Maoz, D., Netzer, H., et al. 2005, ApJ, 629, 61

Kochanek, C. S., Falco, E. E., \& Schild, R. 1995, ApJ, 452, 109

Miralda-Escudé, J., \& Léhar, J. 1992, MNRAS, 259, 31

More, A., Jahnke, K., More, S., et al. 2011, ApJ, 734, 69

More, A., Oguri, M., Kayo, I., et al. 2016a, MNRAS, 456, 1595

More, A., Verma, A., Marshall, P. J., et al. 2016b, MNRAS, 455, 1191

Muñoz, J. A., Falco, E. E., Kochanek, C. S., et al. 1998, Ap\&SS, 263, 5

Myers, S. T., Jackson, N. J., Browne, I. W. A., et al. 2003, MNRAS, 341, 1

Oguri, M., Inada, N., Pindor, B., et al. 2006, AJ, 132, 999

Oguri, M., Inada, N., Clocchiatti, A., et al. 2008, AJ, 135, 520

Ostrovski, F., McMahon, R. G., Connolly, A. J., et al. 2017, MNRAS, 465, 4325

Paraficz, D., Courbin, F., Tramacere, A., et al. 2016, A\&A, 592, A75

Pâris, I., Petitjean, P., Rollinde, E., et al. 2011, A\&A, 530, A50

Pâris, I., Petitjean, P., Ross, N. P., et al. 2017, A\&A, 597, A79

Planck Collaboration XIII. 2016, A\&A, 594, A13

Rhoads, J. E., Dey, A., Malhotra, S., et al. 2003, AJ, 125, 1006

Shen, J., Vanden Berk, D. E., Schneider, D. P., \& Hall, P. B. 2008, AJ, 135, 928

Shen, Y., McBride, C. K., White, M., et al. 2013, ApJ, 778, 98

Shu, Y., Bolton, A. S., Brownstein, J. R., et al. 2015, ApJ, 803, 71

Shu, Y., Bolton, A. S., Kochanek, C. S., et al. 2016a, ApJ, 824, 86

Shu, Y., Bolton, A. S., Mao, S., et al. 2016b, ApJ, 833, 264

Shu, Y., Brownstein, J. R., Bolton, A. S., et al. 2017, ApJ, 851, 48

Smee, S. A., Gunn, J. E., Uomoto, A., et al. 2013, AJ, 146, 32

van der Walt, S., Colbert, S. C., \& Varoquaux, G. 2011, Comput. Sci. Eng., 13, 22

Walsh, D., Carswell, R. F., \& Weymann, R. J. 1979, Nature, 279, 381

Warren, S. J., \& Dye, S. 2003, ApJ, 590, 673

Warren, S. J., Hewett, P. C., Lewis, G. F., et al. 1996, MNRAS, 278, 139

Wayth, R. B., Warren, S. J., Lewis, G. F., \& Hewett, P. C. 2005, MNRAS, 360, 1333

Weymann, R. J., Latham, D., Roger, J., et al. 1980, Nature, 285, 641

Williams, P. R., Agnello, A., Treu, T., et al. 2018, MNRAS, 477, L70

Willis, J. P., Hewett, P. C., Warren, S. J., Dye, S., \& Maddox, N. 2006, MNRAS, 369, 1521

Young, P., Deverill, R. S., Gunn, J. E., Westphal, J. A., \& Kristian, J. 1981, ApJ, 244, 723

Zwicky, F. 1937, Phys. Rev., 51, 290 


\section{Appendix A: QSO-SLE detections}

We present the three first SLEs from the sample of 49 that were not deemed sufficiently asymetric to be considered QSO-LAE candidates. Figure A.1 shows the QSO spectra and the emission line from the SLE. The distribution of the source and lens redshifts for the QSO-SLEs systems is given in Fig. 9, where the redshift of the SLE is computed assuming the emission line is indeed Lyman- $\alpha$ or alternatively O II $3727 \AA$.
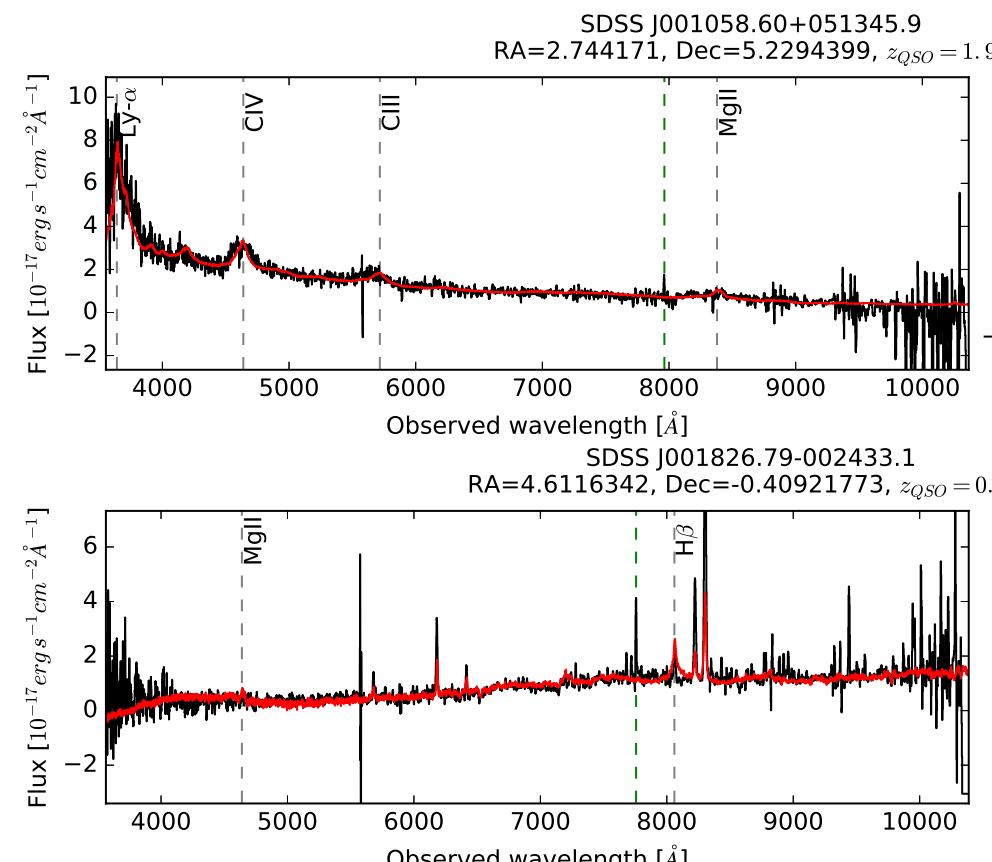

SDSS J011606.17+143916.1

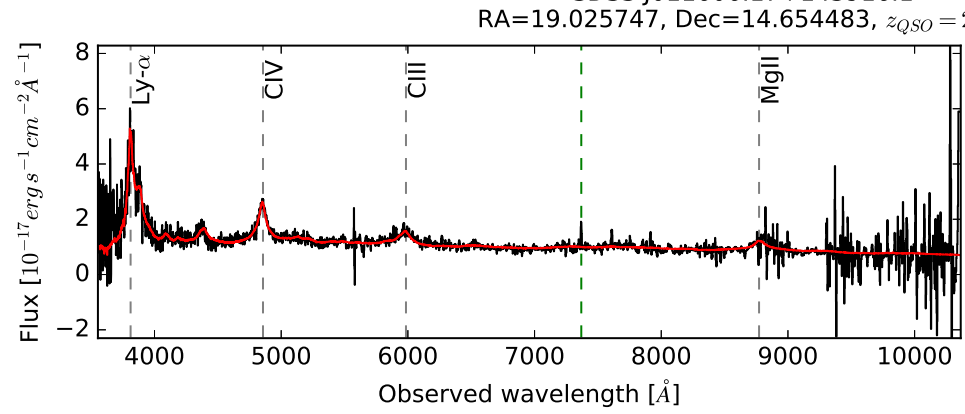

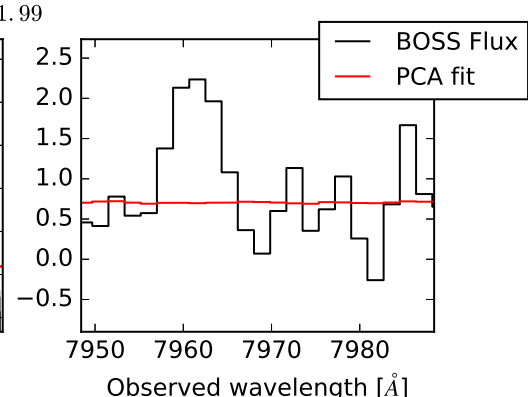

Observed wavelength $[\AA]$

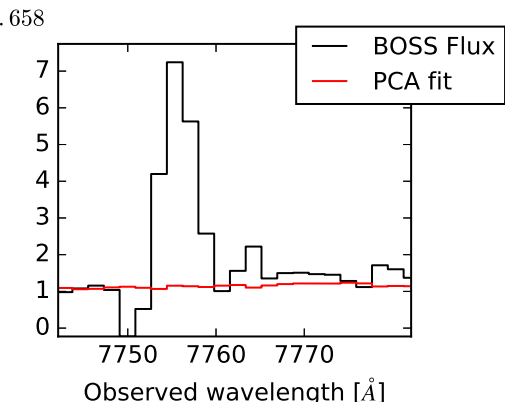

Observed wavelength $[\AA]$

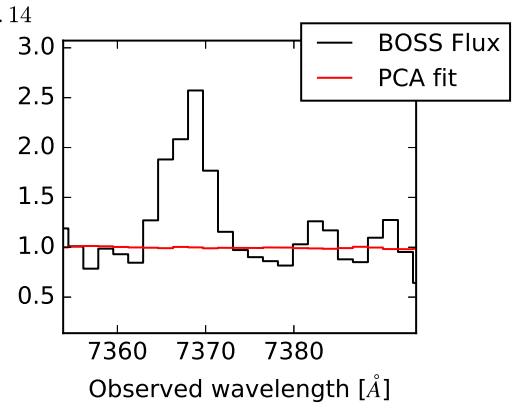

Fig. A.1. SDSS spectra of the three first SLEs of the sample. Even though a clear emission line is detected in each QSO spectrum. Emission lines are either not asymmetric enough to qualify as a potential Lyman- $\alpha$ or appear in noisy regions of the spectra. 


\section{Appendix B: Masked QSO emission and sky emission lines}

We report the masked regions of the observed SDSS spectra due to QSO emission lines (Table B.1) and common sky lines B.2. In each case, we give the central rest-frame wavelength and the rest-frame width of the masked region in Angströms. We note that the observed width of the masked regions increases with redshift and diminishes the search space for supplementary emission lines, as discussed in Sect. 4.

Table B.1. Masked QSO emission lines (see Sect. 2).

\begin{tabular}{lrr}
\hline \hline Denomination & Wavelength $(\AA)$ & Mask width $(\AA)$ \\
\hline Lyman- $\alpha$ & 1215.57 & 300 \\
N V 1240 & 1240.81 & 25 \\
SiIV + OIV 1400 & 1399.80 & 75 \\
C IV 1549 & 1549.48 & 100 \\
He II 1640 & 1640.42 & 50 \\
C III] 1908 & 1908.73 & 50 \\
C II 2326 & 2326 & 25 \\
Ne IV 2423 & 2423.83 & 50 \\
Mg II 2799 & 2799.49 & 125 \\
Ne V 3347 & 3346.79 & 25 \\
Ne V 3427 & 3426.85 & 25 \\
[O II] 3727 & 3727 & 50 \\
[Ne III] 3868 & 3868.75 & 25 \\
H $\delta$ & 4101.73 & 25 \\
H $\gamma$ & 4340.46 & 75 \\
Weak Fe & 4490 & 50 \\
H $\beta$ & 4861.325 & 50 \\
[O III] 4959 & 4958.91 & 100 \\
[O III] 5007 & 5006.84 & 100 \\
Weak Fe & 5080 & 50 \\
N I 5200 & 5200.53 & 50 \\
Weak Fe & 5317 & 50 \\
Weak Fe & 5691 & 50 \\
[Fe VII] 5722 & 5722.30 & 50 \\
[Fe VII] 6087 & 6087.98 & 25 \\
Weak Fe & 6504 & 50 \\
H $\alpha$ & 6562.80 & 5716.44 \\
[S II] 6716 & 6730.82 & 50 \\
[S II] 6730 & & 50 \\
\hline & & 50 \\
\hline
\end{tabular}

Notes. Masked emission lines and width of the mask. Due to the large widths and the binning of the spectra, the central wavelength is rounded up for the implementation. The most important lines were masked a priori, while the fainter iron features were added a posteriori following the over-densities of false positives in the QSO rest-frame.
Table B.2. Masked sky emission lines (see Sect. 2).

\begin{tabular}{lrr}
\hline \hline Denomination & Wavelength $(\AA)$ & Mask width $(\AA)$ \\
\hline Sky 5577 & 5577 & 20 \\
Sky 5896 & 5895 & 25 \\
Sky 6300 & 6300 & 30 \\
Sky 6363 & 6363 & 30 \\
\hline
\end{tabular}

